\title{
RESEARCH HIGHLIGHT OPEN Dietary intervention, a promising adjunct for cancer therapy
}

Rongchen $\mathrm{Shi}^{1}$ and Hongming Miao ${ }^{1}{ }^{1}$

Signal Transduction and Targeted Therapy (2020)5:160

A recent study by Irene Caffa and colleagues published in Nature demonstrated that fasting-mimicking diet (FMD) could enhance the effect of endocrine therapeutics (ET) tamoxifen and fulvestrant for breast cancer (BC) and reduce their side effects, which might provide a new adjunct to endocrine therapy for $\mathrm{BC}^{1}$.

$B C$ is a serious threat to women's health worldwide ${ }^{2}$. Owing to the expression of estrogen receptor (ER) in $\sim 75 \%$ of patients with $\mathrm{BC}$ and the stimulation of tumor growth by estrogen, one of the current treatment strategies for $\mathrm{ER}^{+} \mathrm{BC}$ is to reduce the concentration of estrogen, also known as endocrine therapy. Unfortunately, $\mathrm{ER}^{+} \mathrm{BC}$ is frequently resistant to tamoxifen and fulvestrant, which are two common endocrine therapy drugs ${ }^{3}$. Now, a new adjuvant therapy has been proved that FMD can promote the response of hormone-receptor-positive breast cancer to ET and enhance the antitumor effect (Fig. 1).

The activation of phosphoinositide 3-kinase (PI3K)-AKTmammalian target of rapamycin (mTOR) axis and mitogenactivated protein kinase by growth factor signaling is one of the main factors resulting in ET resistance in BC. Meanwhile, FMD was found to reduce circulating growth factors such as insulin and IGF1. Therefore, the authors speculated that FMD might reduce the resistance of $B C$ to ET through growth factor signaling.

The results showed that tamoxifen and fulvestrant plus FMD could lower the level of IGF1, insulin and leptin, and inhibit AKTmTOR signaling via upregulating epidermal growth factor 1 , the expression of which is associated with good prognosis in patients with BC, and PTEN, a negative regulator of AKT-mTOR signaling, and ultimately suppress tumor progression in mice.

In addition, FMD and ET also synergistically reduced the expression of cell cycle-associated genes (cyclin D1, etc) and consequently promoted cell cycle arrest in BC. Importantly, authors also found that FMD or palbociclib, a cyclin-dependent kinase 4/6 inhibitor, could postpone the occurrence of fulvestrant resistance to a similar extent. The combination of fulvestrant, FMD, and palbociclib displayed an obviously better antitumor effect.

Interestingly, the authors also found that FMD suppressed tamoxifen-induced endometrial hyperplasia. Clinical trials also showed that the prognosis of most BC patients was improved after adjuvant FMD therapy. Simultaneously, growth factor levels were reduced in almost all participants receiving FMD therapy.

Collectively, according to the mouse and clinical studies, Irene Caffa and colleagues proved that FMD could enhance the antitumor activity of ET by reducing the levels of blood growth factors, such as insulin, IGF1 and leptin, with the subsequent inhibition of the PI3K-AKT-mTOR axis.
; https://doi.org/10.1038/s41392-020-00271-y

Diet-related cancer therapy has received a lot of attention recently. Dietary habits, dietary ingredients, and dietary combinations with drugs may have unexpected effects in different cancers and in different stages of a special cancer. As described by Irene Caffa et al., FMD could directly suppress the growth of hormonereceptor-positive breast cancer cells. In fact, dietary interventions may affect all aspects of the tumor microenvironment, not just tumor cells. For example, the immune status of the tumor microenvironment is an important determinant of tumor progression. Previous studies have found that fasting and severe calorie restriction could affect the levels and function of a variety of immune cells ${ }^{4,5}$. During dietary restriction, the increase in the number of adipocytes and nutritive factors in bone marrow promoted the accumulation of memory $\mathrm{T}$ cells, thus improving

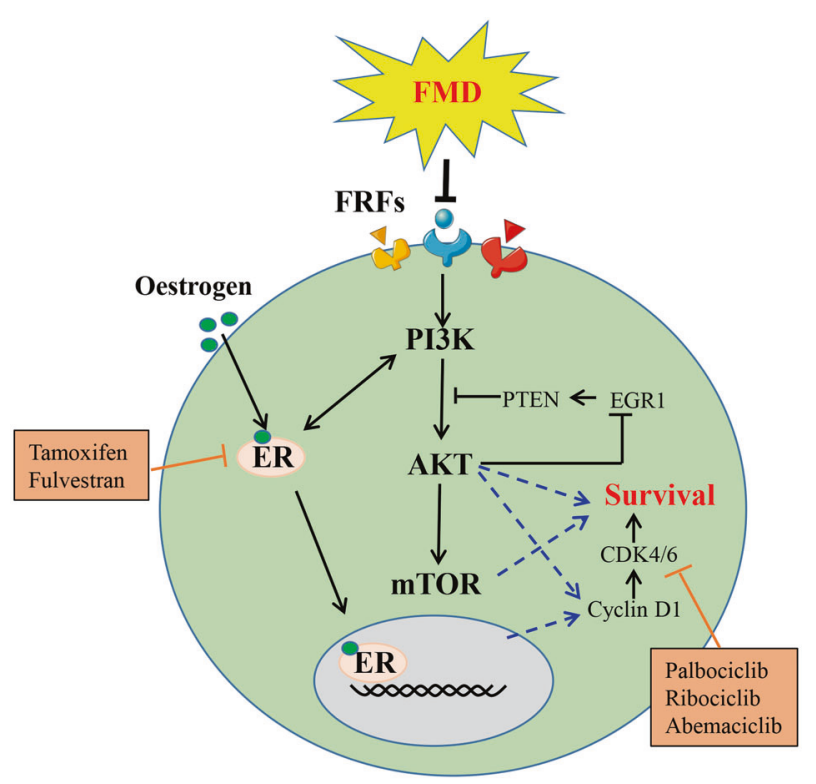

$\mathrm{HR}^{+}$breast cancer cells

Fig. 1 Working model for the cooperation between FMD and ET at the level of PI3K-AKT-mTOR and CCND1. FMD reduces the level of FRFs and inhibits AKT-mTOR signaling via upregulation of EGR1 and PTEN to improve the response of hormone-receptor-positive breast cancer cells to endocrine therapy. AKT inhibition also suppresses the cell cyclerelated genes to cause cell cycle arrest. FMD fasting-mimicking diets, FRFs fasting-reduced factors, such as insulin, leptin, IGF1, ER estrogen receptor, $E T$ endocrine therapy $H R^{+}$hormone-receptor positive 
the resistance to infection and tumors ${ }^{5}$. One of our study also found that high-fat diet treatment in an early phase could prevent peritoneal seeding of colorectal cancer by regulating innate and adaptive immunity (unpublished). Therefore, whether and how FMD would regulate antitumor immunity in BC or other cancers may be an interesting research direction in the future.

Taken together, Irene Caffa et al. suggest that FMD may have significant application potential in cancer therapy. FMD not only increases the sensitivity of $B C$ to existing ET treatment, but also reduces their side effects. Simultaneously, this study also reconfirmed the role of diet intervention in tumor therapy, providing a theoretical basis for larger clinical studies of FMD.

\section{ACKNOWLEDGEMENTS}

This work was supported in part by award numbers 81872028 and 81672693 (H.M.) from the National Natural Science Foundation of China, cstc2017jcyjBX0071 (H.M.) from the Foundation and Frontier Research Project of Chongqing and T04010019 (H.M.) from the Chongqing Youth Top Talent Project.

\section{ADDITIONAL INFORMATION}

Competing interests: The authors declare no competing interests.

\section{REFERENCES}

1. Irene, C. et al. Fasting-mimicking diet and hormone therapy induce breast cancer regression. Nature 583, 620-624 (2020).

2. Harbeck, N. et al. Breast cancer. Lancet 389, 1134-1150 (2017).

3. Waks, A. G. et al. Breast cancer treatment: a review. JAMA 321, 288-300 (2019).

4. Nagai, M. et al. Fasting-refeeding impacts immune cell dynamics and mucosal immune responses. Cell 178, 1072-1087 (2019).

5. Jordan, S. et al. Dietary intake regulates the circulating inflammatory monocyte pool. Cell 178, 1102-1114 (2019).

Open Access This article is licensed under a Creative Commons Attribution 4.0 International License, which permits use, sharing, adaptation, distribution and reproduction in any medium or format, as long as you give appropriate credit to the original author(s) and the source, provide a link to the Creative Commons license, and indicate if changes were made. The images or other third party material in this article are included in the article's Creative Commons license, unless indicated otherwise in a credit line to the material. If material is not included in the article's Creative Commons license and your intended use is not permitted by statutory regulation or exceeds the permitted use, you will need to obtain permission directly from the copyright holder. To view a copy of this license, visit http://creativecommons. org/licenses/by/4.0/.

(c) The Author(s) 2020 